\title{
Major cellular events in peripheral nerve regeneration: A brief overview
}

Naidu $M$ and David $P$

Department of Anatomy, Faculty of Medicine, University of Malaya, 50603 Kuala Lumpur, MALAYSIA

\begin{abstract}
Injury to a peripheral nerve leads to degeneration of the segment distal to the site of lesion, a process referred to as Wallerian degeneration. During Wallerian degeneration, axons and myelin sheaths undergo degeneration and are phagocytosed by macrophages and Schwann cells. The Schwann cells proliferate and the endoneurial tubes persist, together the whole structure is known as the band of Büngner. Within few hours, the damaged axons in the proximal stump initiate a regeneration response, with formation of new growth cones. During Wallerian degeneration, neurotrophins, neural cell adhesion molecules, cytokines and other soluble factors are upregulated to facilitate regeneration. The recovery of the target in mammals is often variable, but almost never complete. In humans, scar tissue forms at the site of lesion and this often results in poor recovery of the target. The major events underlying this regenerative process is highlighted and discussed in this review.
\end{abstract}

KEY WORDS: Wallerian degeneration, Nerve regeneration, Nerve injury

\section{INTRODUCTION}

Peripheral nerve damages such as compression, stretch or traction, friction and even partial or complete transection are not uncommon following traumatic injuries in humans. The outcome of these injuries can range from loss of sensation, formation of painful neuroma, to complete paralysis of the target muscle. Repair of the injured nerves are often poor and the recovery of the affected target muscle tend to be variable. Research carried out over the last few decades focused on understanding the basic mechanism of nerve injury, with a long term hope of improving nerve repair. This paper reviews the major findings in relation to structure and function of various cellular components found in the peripheral nerve during regenerative process following nerve damage. Wallerian degeneration

Following insult to a peripheral nerve, the portion distal to the injury undergoes a process known as Wallerian degeneration. The original description of degeneration of the distal portion of a cut axon, together with the degeneration of its myelin sheath came from experiments conducted by Augustus Waller (1850) cited after Richardson and De Girolami. ${ }^{1}$ This phenomenon has been extensively studied since; the

Correspondence author:

Pamela David, Department of Anatomy,

Faculty of Medicine,

University of Malaya,

50603 Kuala Lumpur,

Malaysia.

E-mail: rosiepamela@um.edu.my most cited early work being that of Ramony Cajal. ${ }^{2}$

When a peripheral nerve is cut, there is an immediate paralysis of the muscles and loss of sensation in the area supplied by the nerve. Nevertheless, the axon would remain intact for 3 to 5 days in humans and primates, and possibly less in rodents. In the distal stump, the cytoskeletal structures (neurofilaments and microtubules) go through disintegration, with accumulation of granular debris in the axoplasm. The cell membrane disappears, the axon undergoes fragmentation and the myelin rapidly disintegrates. In rodents, these events usually occur between 2 to 3 days following axotomy.

Following the breakdown of axons and myelin, the Schwann cells in the distal stump undergo changes. Within the first two weeks following axotomy, there is an increase in Schwann cell processes and they proliferate longitudinally within the tubes formed by the intact basal lamina. Eventually, these divided Schwann cells within the tubes of basal lamina, form long cellular chains and the whole structure is known as the band of Büngner which plays an important role in peripheral nerve regeneration. ${ }^{1}$

Apart from Schwann cells, the macrophage population also increases in the degenerating nerve. In rats, invasion of macrophages usually occur within 24 hours of injury. Usually, there are a few macrophages in the walls of the endoneurial blood vessels, but following nerve injury; most of the macrophages are recruited from the bloodstream. Over the next 1 to 2 days, the number of macrophages increases tremendously and they pass through the intact Schwann cell basal laminae and phagocytose the remains of the myelin and axons. Within the next one to two weeks, much of the debris is cleared from what have become the bands of 
Büngner. Even though macrophages are the main cells responsible for myelin phagocytosis, there are other studies that have shown the presence of myelin debris within the Schwann cells indicating that Schwann cells are also capable of ingesting myelin. ${ }^{3}$ In summary, the main aim of the Wallerian degeneration is to remove axon- and myelin-derived material to pave way for the regenerating axons.

\section{Cell body reaction to nerve injury}

The most consistent and conspicuous observation in cell body following nerve injury is probably chromatolysis, which involves the disintegration of the Nissl substance (large condensation of the endoplasmic reticulum). In addition, nuclear eccentricity, nucleolar enlargement and cell swelling can be clearly noted. Other changes include increases in cytoplasmic acid phosphatase and smooth endoplasmic reticulum with hypertrophy of Golgi apparatus. ${ }^{4}$ The highlight of the metabolic event seems to be an increase in the nuclear RNA synthesis, which is associated with an increase in cytoplasmic protein synthesis and content. ${ }^{5}$

A study by Martin et al, indicated that chromatolysis is the same as apoptosis. ${ }^{6}$ Their study showed that by 21 days following unilateral sciatic nerve avulsion in adult rats, the number of large motoneurons in the lumbar spinal cord was reduced by approximately $30 \%$. The investigators found that the dying motoneurons were positively stained for the terminal transferasemediated deoxyuridine triphosphate biotin nick-end labelling which detects fragmentation of nuclear DNA during apoptosis. These investigators also reported that the dying motoneurons undergo chromatolysis followed by progressive cytoplasmic and nuclear condensation with chromatin compaction into uniformly large round clumps. In addition, by using immunodetection method, the investigators also found that these dying motoneurons sustain oxidative damage to proteins and nucleic acids within the first 7 days after injury during the progression of apoptosis.

Proteins such as growth associated proteins (GAPs), tubulin and actin have been reported to be enhanced during regeneration, whereas neurofilament protein, which is mainly expressed in mature axons, is decreased. In addition, two of the tubulin genes, T alpha 1 and class II beta are also activated during regeneration. ${ }^{7,8}$

c-Jun is a protein that may be important for successful axon regeneration. Studies by Broude et al, on adult rat dorsal root ganglia (DRG) showed that c-Jun was substantially upregulated in DRG neurons following a peripheral axotomy, but after a central axotomy, only $18 \%$ of the neurons expressed c-Jun. ${ }^{9}$ Similar strong up-regulation of c-Jun in the adult rat DRG following peripheral nerve transection was also reported earlier by Leah et al. ${ }^{10}$ However, the events that link c-Jun up-regulation to regeneration are yet to be identified.
Two axonally transported cystoskeletal proteins that are induced very rapidly following axotomy are GAP43 and CAP23 which are involved in actin cytoskeleton and neurite outgrowth. Others have shown that these two proteins are functionally related and play a critical role in regulating nerve sprouting and the actin cytoskeleton. ${ }^{11}$ Their studies on knockout mice lacking CAP23, exhibited pronounced and complex phenotype defects including inability to produce stimulusinduced nerve sprouting at the adult neuromuscular junction. These authors reported that this sprouting deficit was rescued by transgenic over expression of either CAP23 or GAP43 in the motoneurons. The investigators also observed that knock-in mice expressing GAP43 instead of the CAP23 were essentially normal and concluded that although these proteins do not share homologus sequences, GAP43 can functionally substitute CAP23 in vivo. Additionally, the investigators also found that cultured sensory neurons lacking CAP23 exhibited striking alterations in neurite outgrowth.

Another secretory protein, which is known as Reg-2, has been shown to promote regeneration in the sensory and motor neurons after peripheral nerve injury in rats. Reg- 2 appears to be massively up-regulated in the subsets of sensory neurons and in all regenerating a motor neurons after sciatic nerve crush. ${ }^{12}$ Reg-2 seems to have mitogenic affect on Schwann cells and following injection of Reg-2 antibody into the crushed nerve, axon regeneration in the relevant neurons appear to be retarded. Reg- 2 is also reported to be expressed in a subpopulation of motoneurons and this expression is driven by cytokines which have been shown to prevent cell death, such as interleukin-6 (IL-6), ciliary neurotrophic factor (CNTF), leukaemia inhibitory factor (LIF) and cardiotrophin (CT-1). ${ }^{13}$ However, the role of Reg- 2 expression in the sensory neurons is yet to be elucidated.

Bonilla et al, reported that a protein called small proline-rich repeat protein 1A (SPRR1A) is highly induced in injured dorsal root ganglia neurons and axons following peripheral nerve damage. ${ }^{14}$ They also found that over-expressed SPRR1A co-localised with actin in membrane ruffles and augmented axonal outgrowth. In addition, the investigators also found that reduction of SPRR1A function restricts axonal outgrowth and concluded that neuronal SPRR1A may be a significant protein contributing to successful nerve regeneration.

In contrast to the response of adult nerve cell body discussed above, peripheral nerve injury in the neonatal animals such as rats, results in large proportion of the motoneurons dying. ${ }^{15}$ The reason for this is thought to be that these young developing neurons are deprived of target-derived and retrogradely-transported neurotrophic support following nerve injury at a time when they do not have access to other forms of trophic support, and are particularly vulnerable. 
From the injured end of the axons, regenerative sprouts begin to appear within 20 hours following axotomy. These sprouts are multiple and a single axon can give rise to as many as 25 sprouts. The fate of these sprouts is very much dependent on the proximity to the distal stump of the nerve and the extent of collagenous scar tissue developed at the injury site. If the cut ends are approximated with little intervening scar, many of the regenerating axons can cross the gap and enter the endoneurial tubes found in the distal stump. However, if a wider gap exists between the proximal and distal stump, or if scar tissue forms in this gap, the regenerating axons will be prevented from reaching the distal stump and these axons would turn back and form tangle masses, resembling amputation neuromas. This phenomenon was well documented by Cajal. ${ }^{2}$

Those of the multiple axonal sprouts that reach the distal stump enter the endoneurial tubes, forming clusters of axons. Gradually, some of the axons enlarge and reach the normal diameter of a parent axon. However, many of the axonal sprouts retract probably because they do not get to a target. Meanwhile, the Schwann cells in the endoneurial tube rearrange to form a one-to-one relation between the Schwann cells and axons. Eventually, the Schwann cells remyelinate the axons that have attained a certain critical size. ${ }^{2,16,17}$

On the other hand, if the epineurium is still intact as in a nerve crush, compression or ischemic injuries, the pathway for regeneration is relatively intact and axon regeneration under these circumstances is much more favourable and the regenerating axons would be guided back to their original target more accurately. Studies carried out on the adult rat common peroneal nerve showed that, following a nerve crush where the epineurium is still intact, the regenerating axons were seen contacting the motor endplates, in this case motor endplates of the extensor digitorum longus muscle, between 7 and 14 days after injury. 18 By using the silver cholinesterase staining method, the authors also reported that by day 14 after injury, $89 \%$ of the motor endplates were re-innervated and resulted in good functional recovery of the target muscle.

\section{CONCLUSION}

So far, research carried out on the peripheral nerve and its regeneration process have identified many of the structures and components involved, and also elucidated basic underlying mechanism behind the regeneration process. However, our understanding of the exact biology of the peripheral nerve regeneration in particular the environmental influences and axonal mechanisms are still far from complete and future studies should focus in theses areas.
I would like to thank Professor James Fawcett, Cambridge Centre for Brain Repair, University of Cambridge, England, and United Kingdom for his invaluable comments during the preparation of this manuscript.

\section{REFERENCES}

1. Richardson EP, De Girolami U. Pathology of the peripheral nerve. In: General reactions of peripheral nerve to disease. Philadelphia: Saunders, 1995:8-21.

2. Cajal S. Degeneration and regeneration of the nervous system. Translation by May RM, London: Oxford University Press, 1928.

3. Fernandez Valle C, Bunge RP, Bunge MB. Schwann cells degrade myelin and proliferate in the absence of macrophages: evidence from intro studies of Wallerian degeneration. J Neurocytol 2002; 24:667-9

4. Lieberman AR. The axon reaction: a review of the principal features of perikaryal responses to axon injury. Int Rev Neurobiol $1971 ; 14: 49-124$

5. Lieberman AR. Comments on the fine structural organization of the dorsal lateral geniculate nucleus of the mouse. Anat Em bryol (Berl) 1974; 145:261-7

6. Martin LJ, Kaiser A, Price AC. Motor neuron degeneration after sciatic nerve avulsion in adult rat evolves with oxidative stress and is apoptosis. J Neurobiol 1999; 40: 185-201

7. Fawcett JW, Keynes RJ. Peripheral nerve regeneration. Annu Rev Neurosci 1990; 13:43-60

8. Snider WD, Zhou FQ, Zhong J, Markus A. Signaling the pathway to regeneration. Neuron 2002; 35:13-6

9. Broude E, McAtee M, Kelley, MS, Bregman BS. c-Jun expression in adult rat dorsal root ganglion neurons: differential response after central or peripheral axotomy. Exp Neurol 1997; 148:367-77

10. Leah JD, Herdegen T, Bravo R. Selective expression of Jun proteins following axotomy and axonal transport block in peripheral nerves in the rat: evidence for a role in the regeneration process. Brain Res 1991; 566:198-207

11. Frey D, Laux T, Xu L, Schneider C, Caroni P. Shared and unique roles of CAP23 and GAP43 in actin regulation, neurite outgrowth, and anatomical plasticity. J Cell Biol 2000; 149:1443-54

12. Averill S, Davis DR, Shortland PJ, Priestley JV, Hunt SP. Dynamic pattern of reg-2 expression in rat sensory neurons after peripheral nerve injury. J Neurosci 2002; 22:7493-7501

13. Livesey FJ, O'Brien JA, Li M, Smith AG, Murphy LJ, Hunt SP. A Schwann cell mitogen accompanying regeneration of motoneurons. 
Nature 1997; 390:614-8

14. 14. Bonilla IE, Tanabe K, Strittmatter SM. Small proline-rich repeat protein $1 \mathrm{~A}$ is expressed by axotomized neurons and promotes axonal outgrowth. J Neurosci 2002; 22:1303-15

15. Lowrie MB, Krishnan S, Vrbova G.

Permanent changes in muscle and motoneurones induced by nerve injury during a critical period of development of the rat. Brain Res 1987; 428:91-101

16. Lundborg G. Nerve injury and repair. Edinburgh: Churchill Livingstone, 1988:199204.

17. Sunderland S. Nerves and nerve injuries. Part 1: Peripheral Nerve Fibers. New York; Churchill Livingstone, 1978:3-20.

18. Naidu MDK, Subramaniam K, Vrbovä G. Re-innervation of muscles after nerve injury in neonates. Restor Neurol Neurosci 1996; 10:35-42 